\title{
Evolution of Equity Market Risk During the Crisis: Europe, Americas and Asia
}

\author{
Patrizia Stucchi • Giorgio Dominese
}

Published online: 23 November 2012

(C) Springer-Verlag Wien 2012

\begin{abstract}
A set of regional and country's equity indices have been evaluated and analysed in their Value at Risk (VaR) and Conditional Value at Risk (CVaR) in this paper, using computational methods based on the Johnson systems. Comparing the main statistics and the values of the two cited measures of financial risk obtained using a roll-over mechanism in the period January 2008-July 2012, the impact of the crisis on equity market risk can be shown. It seems that for all regions and countries the patterns are very similar: there is a peak of all the risk measures adopted at the beginning of the crisis (September 2008-February 2009) and another turbulent period in 2011 (from July to December). In other terms, the global patterns of the main financially relevant countries and their regional aggregations demonstrate that "One Financial system", and just one, is already at work, in theory and in practice. On the other hand, the scale of the risk measures differs from one country to another: e.g., with a probability of $1 \%$, the potential daily loss on an equity position in Latin America in the worst period arrives to about $25 \%$, the Emerging Markets as a whole show values around $20 \%$ and Asia arrives to $15 \%$, while the US and European corresponding values are below $14 \%$. This is true whatever the risk measure and whatever the confidence interval (which, again, influences strongly the scale of the risk values). Looking in detail to the last period (April 2012-July 2012), a general improvement could be appreciated: the risk measures are all around
\end{abstract}

Published with the permission of Transition Studies Review.

P. Stucchi $(\bowtie)$

Department of Economics and Statistics, University of Udine, Udine, Italy

e-mail: patrizia.stucchi@uniud.it

G. Dominese

School of Economics, University of Tor Vergata, Rome, Italy

e-mail: giorgio.dominese@uniroma2.it; gdominese@luiss.it

G. Dominese

School of Political Sciences, LUISS University, Rome, Italy 
$4 \%$ if not on one hand Italy and Spain (around $6 \%$ ), Greece (around $10 \%$ ) and on the other hand the "virtuous" Chile (around $1.5 \%$ ), again with reference to a probability of $1 \%$. Nevertheless, indices of performance (expected return over risk measure) have been evaluated and compared. They give sometimes different answers to the risk measures themselves.

Keywords Country equity conditional value at risk - Financial crisis . Risk measures · Expected shortfall $\cdot$ Skewness $\cdot$ Kurtosis

JEL Classification $\mathrm{G} 32 \cdot \mathrm{G} 01 \cdot \mathrm{F} 30 \cdot \mathrm{C} 63$

\section{Introduction}

Financial equity risk of a country may be measured in terms of Value at Risk or Conditional Value at Risk of a representative financial stock index. The main purpose of the paper is to investigate the evolution of these measures over the period January 2008-July 2012 and to analyze the relevance of tail risk. In case of financial market instability, the classical measures of riskiness such as the volatility are not fully reliable, because the probability of extreme events is not negligible. The risk measure suitable to capture tail risk is the expected shortfall (or Conditional Value at Risk$C V a R$ - under regularity conditions on distributions). The evaluation of CVaR requires previously the estimation of quantiles (substantially $V a R$ in financial applications) and usually $C V a R$ may be found using numerical evaluation procedure (there are no closed formulas). Here a transformation based on the first four moments (Johnson systems, 1949; 1965) has been adopted in the empirical investigations. The Johnson systems may be applied to a wide set of random variables, also when critical situations of financial markets give rise to heavy-tailed distribution with high kurtosis.

Moreover, applying the Johnson systems allows to obtain closed or quasi-closed formulas for CVaR calculation (see Simonato 2011). It is worthwhile to underline that the Johnson's approach requires only the estimation of four moments in order to find $C V a R$ and this represents a very appealing feature from a practical point of view.

With the aim of analyzing and comparing the evolution of all these risk measures, we adopt a roll-over evaluation mechanism over the period 2008-2012, obtaining for any index, for any risk measure and for five different levels of probability about a thousand of risk measures' daily values.

This procedure has been applied to historical data relative to 27 stock indices of emerging and European markets.

We have also considered and compared measures of performance of equity markets (expected return over measure of risk).

In the following section the definitions of $V a R$ and $C V a R$ have been recalled in detail, together with the basic notations and the moments' method by Johnson. In the same section the Johnson systems closed-formulas for VaR and $\mathrm{CVaR}$ have been described. The section with the numerical results includes the analysis of values of $\mathrm{VaR}$ and $\mathrm{CVaR}$ obtained using the Johnson's approach and also the evaluation and the analysis of the performance measures. The last section contains the conclusions and comments. 


\section{Measures of Risk}

\section{VaR and $C V a R$}

Starting from the random variable loss $L$ of a position over a time horizon $[t, t+\tau]$, a measure of risk is the critical level of loss corresponding to a probability $c$. This critical level is named VaR (Value at Risk) and formally it corresponds to the generalized $c$-quantile of $L:^{1}$

$$
\operatorname{VaR}=F_{L}^{\leftarrow}(c) \text {. }
$$

For example, considering $c=99 \%$ and a daily horizon, VaR represents the worst potential loss on the position with probability $1 \%$ within $24 \mathrm{~h}$.

In terms of the random variable return $R$ on a position, it is possible to show that $V a R$ may be evaluated using the following condition:

$$
\operatorname{VaR}=-w \cdot F_{R}^{\leftarrow}(1-c)
$$

where $w$ is the initial value of the position. Considering an initial unit value $(w=1)$, VaR becomes a percentage measure of risk or, in other words, the percentage critical loss with probability $c$ on the fixed time horizon. Hereafter this definition of $V a R$ will be used.

The most important critique to $V a R$ is linked to the idea that risk measures must satisfy coherence properties (Artzner et al. 1999). Briefly, these properties are:

(T) Translation invariance: adding a risk free investment (or liquidity) to the old position will reduce the value of the risk measure;

(H) Positive homogeneity: if we sum up $\lambda$ positions of the same kind, all characterized by the same (kind of) loss $L$, the global risk is $\lambda$ times the risk on a single position;

(S) Subadditivity: the risk on a sum of (different) losses must be less than the sum of the risks on losses separately considered. This means substantially that building portfolios of positions composed by different random losses grants risk diversification;

(M) Monotonicity: this property reflects the idea that if the loss on a position has values not greater than those of loss on another position in every state of the world, then the first position must be less risky than the second.

Unfortunately, it can be shown that VaR satisfies the properties $(\mathrm{T}),(\mathrm{H})$ and $(\mathrm{M})$, but, in general, it suffers from the lack of subadditivity.

Another important measure of risk satisfying all coherency properties is the so called Expected Shortfall (ES) defined as follows:

$$
E S_{c}=\frac{1}{1-c} \int_{c}^{1} F^{\leftarrow}(t) d t .
$$

\footnotetext{
1 The symbol "६" in the definition of $V a R$ has just the meaning of generalized inverse of the considered function; if the function $F$ admits an inverse, the symbol " $\leftarrow$ " becomes " -1 ".
} 
Under regularity conditions over the distribution function it is possible to show that for any probability $c$ belonging to $(0,1)$ the expected shortfall is equal to the Conditional Value at Risk $(C V a R)$, formally:

$$
E S_{c}=E\left(L / L>F^{\leftarrow}(c)\right)=C V a R=E(L / L>V a R) .
$$

The last expression means that for regular distributions $C V a R$ is the expected loss conditioned to worst loss values (exceeding $\mathrm{VaR}$ ). Again, in terms of returns of a position with unit initial value $C V a R$ may be interpreted as the expected return conditioned to the worst return values (below to the opposite of percentage VaR).

\section{Moment Based Quantiles for Unknown Distributions: The Johnson Systems}

In many practical situations, in particular dealing with portfolios containing derivatives, it is impossible to identify exactly the kind of distribution of the stochastic loss or return on the position. This means that it is impossible, at the same time, to find the return cumulative distribution function, the inverse of the cumulative distribution function $(C D F)$, quantiles and $V a R$. If it is possible to estimate the main moments, there are methodologies that provide a proxy distribution based on transformations of normal variables distribution. This kind of approach is called moments' method.

If $X$ is the random variable to be transformed, the characterizing moments and the most important (moment based) indices to be estimated are the expected mean, the standard deviation, the skewness index and the kurtosis index. The Johnson systems approach is based on the idea that the previous parameters may characterize sufficiently well a lot of distributions and that any random variable $X$ may be well approximated in terms of a standard normal variable by a function of the following kind:

$$
X \cong A+B \cdot h\left(\frac{N-C}{D}\right)
$$

where $N$ is a standard normal variable and $A, B, C, D$ are parameters obtained in terms of the moments of $X$. The function $h(\cdot)$ is a non-decreasing monotonic function independent by the variable's moments (with positive $B$ and $D$ ). The choice of the function $h(\cdot)$ is linked to the values of the skewness and kurtosis indices. Let $\beta_{1}$ be the squared skewness and $\beta_{2}$ the kurtosis index. Johnson considers the $\left(\beta_{1}, \beta_{2}\right)$ plane and determines three zones (see Fig. 1).

The simplest form of Johnson's transformations, is denoted by $S_{L}$ :

$$
h(\cdot)=\exp \left(\frac{N-C}{D}\right) .
$$

This implies that the natural logarithm of $(X-A) / B$ follows a log-normal distribution. The log-normal curve in the $\left(\beta_{1}, \beta_{2}\right)$ plane divide the feasible area in two zones: the upper corresponds to the unbounded transformation $S_{U}$ and the lower to the bounded transformation $S_{B}$ (see again Figure 1). The name unbounded and bounded are related to the feasible value of $X$ in the two systems: in the unbounded $X$ may have any real value while in the bounded $X$ may assume values in the 


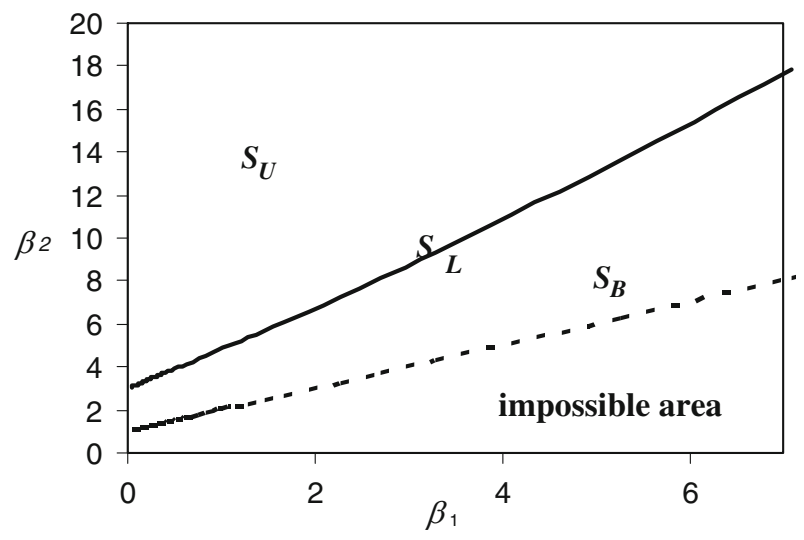

Fig. 1 Johnson Systems

Table 1 VaR in the Johnson Systems

\begin{tabular}{ll}
\hline Johnson transformation & $h(\cdot)$ \\
\hline Log-normal $S_{L}$ & $\exp \left(\frac{N-C}{D}\right)$ \\
Bounded $S_{B}$ & {$\left[1+\exp \left(-\frac{N-C}{D}\right)\right]^{-1}$} \\
Unbounded $S_{U}$ & $\sinh \left(\frac{N-C}{D}\right)$ \\
\hline
\end{tabular}

interval $(A, A+B)$. The functional form $h(\cdot)$ suggested by Johnson are summarized in Table 1.

Once identified the kind of transformation on the basis of the estimated values of the pair $\left(\beta_{1}, \beta_{2}\right)$, an estimation of the four parameters $A, B, C$ and $D$ is needed. It is not simple and not always possible to find a solution matching the input data (the moments). ${ }^{2}$ In the numerical applications the unfavourable conditions giving rise to no solution may happen in particular with reference to situations with both high skewness and high kurtosis. The Johnson moments' method cannot be applied and he suggested alternative method such as a quantile approach. In these cases the Shapiro and Slifker (1980) method may be applied in order to find the parameters of Johnson translation.

VaR and CVaR in the Johnson framework

Exploiting the idea of Johnson, it is possible to transform a wide set of random variables $X$ in function of a standard normal variable with a very good degree of precision. Moreover, it is possible to find closed or quasi-closed formulas for $C V a R$ once found the kind of transformation and the parameters in the Johnson framework

\footnotetext{
${ }^{2}$ A numerical procedure is given by Hill (Griffiths-Hill Archive 1976) and Hill et al. 1976. In the applications a Visual Basic version of these routines has been used, completed with numerical integration routines required in the bounded case.
} 
Table 2 CVaR in the Johnson Systems

\begin{tabular}{lll}
\hline JSN & VaR & CVaR \\
\hline$S_{L}$ & $-\exp \left(\frac{z-C}{D}\right)=-\exp (m+s z)$ & $-\frac{\mu}{1-c} F_{N}(z-s)$ \\
$S_{B}$ & $-A-B\left[1+\exp \left(-\frac{z-C}{D}\right)\right]^{-1}$ & $-A-\frac{B}{1-c} \frac{1}{\sqrt{2 \pi}}\left[\int_{-\infty}^{z} \frac{e^{-u^{2} / 2}}{1+\exp \left(-\frac{u-C}{D}\right)} d u\right]$ \\
$S_{U}$ & $-A-B \sinh \left(\frac{z-C}{D}\right)$ & $-A-\frac{B}{2(1-c)}\left[\exp \left(\frac{1-2 C D}{2 D^{2}}\right) F_{N}\left(z-\frac{1}{D}\right)-\exp \left(\frac{1+2 C D}{2 D^{2}}\right) F_{N}\left(z+\frac{1}{D}\right)\right]$ \\
\hline
\end{tabular}

(see Simonato 2011). Value at Risk and CVaR may be all expressed in the Johnson framework in terms of the $(1-c)$-quantile of a standard normal variable, that is in terms of:

$$
z=F_{N}^{-1}(1-c)
$$

where $F_{N}^{-1}(\cdot)$ is the inverse of the cumulative distribution function of the standard normal variable.

Table 2 summarizes the main results.

In the log-normal Johnson transformation the parameter $A$ value is zero and that of $B$ is 1 and VaR may be expressed in terms of the parameters $m$ and $s$ (mean and standard deviation) of the normal variable corresponding to $\ln (X)$. The log-normal $C V a R$ depends on the estimated mean of $X$, on the fixed level of probability $c$ and on the above cited parameter $s$.

In the bounded transformation $V a R$ is given by a closed formula while $C V a R$ must be evaluated using numerical procedures, while in the unbounded system there are closed formulas for both $V a R$ and $C V a R$.

\section{Numerical Results}

\section{Data and Main Statistics}

Daily data from free databases ${ }^{3}$ have been used. The historical quotes are referred to a set of 27 stock indices of six world regions (World, US, Europe, EEM (Emerging Markets), Asia, Latin America) and 21 countries (China, India, Japan, Korea, Malaysia, Singapore, Sri Lanka, Taiwan, Argentina, Brazil, Chile, Mexico, France, Germany, Greece, Ireland, Italy, Portugal, Russia, Spain, United Kingdom). Data are referred to the period January 2008-July 2012, covering the whole dramatic period of crisis, except for Portugal (June 2010-July 2012). We have considered global stock market indices. Indeed, the distinction between the stocks accessible to both foreign and domestic investors (investable) and those available only to domestic (non investable), summing up to the global value of the index, is very important and should be analyzed further (see Li and Rose 2009).

\footnotetext{
3 Yahoo!@Finance (http://finance.yahoo.com/) supplies historical data on the indices used in this work; close prices adjusted for dividends and splits have been considered.
} 
The database consists of more than one thousand daily prices for any index. The logarithmic returns have been evaluated and the estimation of return moments has been made using the basic standard definitions, that is:

$$
\begin{aligned}
& \text { Mean }=\bar{x}=\frac{1}{N} \sum_{i=1}^{N} x_{i} \\
& \text { Variance }=\bar{\sigma}^{2}=\frac{1}{N-1} \sum_{i=1}^{N}\left(x_{i}-\bar{x}\right)^{2} \\
& \text { Skewness }=\frac{N}{(N-1)(N-2)} \frac{1}{\bar{\sigma}^{3}} \sum_{i=1}^{N}\left(x_{i}-\bar{x}\right)^{3} \\
& \text { Kurtosis }=\frac{N(N+1)}{(N-1)(N-2)(N-3)} \frac{1}{\bar{\sigma}^{4}} \sum_{i=1}^{N}\left(x_{i}-\bar{x}\right)^{4}
\end{aligned}
$$

with $x_{i}$ daily observed log-return and $N$ number of the observations. On the following website http://www.dies.uniud.it/index.php/ricerca-stucchi.html the interested readers will find the link to the file named Datastat containing the summary statistics and the corresponding evaluation of the Johnson parameters, together with the estimated risk and performance measures. For all the sets of moments, the kind of Johnson translation is $S_{U}$ (unbounded) or $S_{B}$ (bounded).

It is worthwhile to observe that values of kurtosis exceeding 20 (very high with reference to the realized values in the database) are rare and perhaps anomalous: they occur three times (two for Malaysia and one for Sri Lanka) on about 27,000 estimations. Also kurtosis values in the interval [10, 20] occur few times (Korea in December 2009, Malaysia and Sri Lanka in March and April 2008, again Sri Lanka in June and July 2009, Italy and Spain in May 2010). The most of values belong to the interval $[2,10]$. Indeed, this means that turbulent period may be characterized by relatively small values of kurtosis. Moreover, it seems that the first period of the crisis not necessarily has implied fat tailed distributions of return on stock indices, but this feature has been showed in other more "quiet" periods. Last, the high values of the risk measures in the end of 2008 and of beginning 2009 corresponds not to high values of kurtosis but to "unfavorable" combinations of skewness and kurtosis.

\section{VaR and CVaR of Emerging and European Countries}

Johnson $\operatorname{VaR}$ and $C V a R$ have been evaluated using formulas in Table 2 for five values of probability $c$, that is $99.99,99.95,99,95$ and $90 \%$. With the aim of analyzing for any index the evolution of equity countries riskiness over the period January 2008-July 2012, we have used a rollover mechanism: using windows of 20 working daily returns (about 1 month) the running moments have been evaluated. This means that, for any index, we have about one thousand values of moments

Observing the results, whatever the measure of risk adopted and whatever the country, it is strongly evident the impact of the crisis in 2008. From May 2009 until July 2011 there is a riskiness reduction almost to levels preceding the crisis, apart 
from the summer 2010 when things were slightly worse. Since the second half of 2011, risk has remained restrained for India, Brazil and Russia while it is growing up for China, the United States and Europe. The graphs reported at the end of the paper show the evolution of Johnson $C V a R$ with probability $\mathrm{c}=99 \%$ for all the indices together with a "zoom" of the last period (from April, 1st 2012 to July, 15th 2012): these graphs represent a synthesis of what happens in terms of country equity risk during the crisis. Whatever the risk measure and the confidence level $c$, the shape of the evolution remains the same: there is only a change in the scale of the values.

Daily risk is quite large both in terms of $\operatorname{VaR}$ and in terms of CVaR: e.g. with probability $(1-c)=1 \%$, the minimum $\operatorname{VaR}$ value is that of Chile (minimum $\operatorname{VaR}=0.13 \%)$ and it is the same for the minimum value of $C \operatorname{VaR}(0.15 \%)$. The maximum values are 26.74 and $37.12 \%$ respectively for Sri Lanka VaR and CVaR over the whole period with $(1-c)=1 \%$. This means that if we have a position of $\$ 1$ million in the Chilean stock market, the potential loss, with probability $1 \%$, in which we may incur in the next $24 \mathrm{~h}$ is $\$ 1,300$ (VaR) while the daily expected loss beyond $\operatorname{VaR}$ is $\$ 1,500(\mathrm{CVaR})$. If we have a position of $\$ 1$ million in the Sri Lanka stock market, the potential loss, with the smaller probability $0.01 \%$, should be $\$ 267,400(\mathrm{VaR})$ over the next $24 \mathrm{~h}$, while the daily expected loss beyond $\mathrm{VaR}$ is $\$ 371,200(\mathrm{CVaR})$.

Considering the minimum and maximum values of $V a R$ and $C V a R$ for all the adopted values of probability over the whole period: this gives evidence of the different scale changing the probability and of the possible differences between $\mathrm{VaR}$ and $C V a R$ (see the above cited link).

In general, differences between daily $V a R$ and $C V a R$ are not negligible and may arrive to more than $20 \%$. This confirms the idea that investors and regulators, in order to evaluate capital requirements, must refer to measures of risk considering the worst part of the distribution especially in presence of unpredictable turbulences in the financial markets.

The estimation of the mean and standard deviation of all the risk measures in every year have been computed. With reference to these values, it is interesting to note that in 2008, the region and country indices having the highest expected value of the risk measures are Latin America, Russia, the Emerging Markets, Ireland, China and Brazil, while the lowest values are those of Sri Lanka, Singapore and Chile. In this year we observe the worst values. From 2010, things have been evolving: some European countries (Greece in particular) have the higher expected values of the risk measures. On the other hand, looking at the volatility of the risk measures, the European countries (with the exception of Greece) are in the middle, that is the fluctuation range of the risk measures have been mainly convergent.

In any case, there has been an important improvement in the transition from the beginning of the crisis to the last period. In fact, $V a R$ and $C V a R$ has more than halved for almost all the regions and countries analysed. To give evidence of these facts, we have reported at the end of the paper the graphs of $V a R$ and $C V a R$ with $c=99 \%$ over the whole period and over the last period (April 2012-July 2012) for any of the considered stock market indices. 
Comparison of the Performances of Emerging and European Countries

With regard to indices of performance, here we refer to the most famous reward to risk ratio, the Sharpe ratio (Sharpe 1994), that has been used together with the VaR Ratio (see Favre and Galeano 2002; Rachev 2003) and the CVaR Ratio (STARR Ratio) (see Rachev 2003). These ratios have been evaluated here considering always at numerator the estimated mean of return and at denominator, respectively, the standard deviation (Sharpe ratio), VaR (VaR Ratio) and CVaR (CVaR Ratio).

The evaluation of the indices of performance, presents different trends with respect to the indications given by the risk measures themselves. Of course, all the before mentioned peculiarities bring Asian and South American emerging markets to an average better performance compared with European Union countries.

\section{Conclusions}

The paper is focused to measurement of countries' equity risk in terms of Value at Risk or Conditional Value at Risk (CVaR) of a meaningful financial stock index and to investigate the evolution of these measures over the horizon 2008-2012. In case of financial market instability, the classical measures of riskiness, such as volatility, are not fully reliable, but the risk measure better capturing tail risk is the expected shortfall (or $C V a R$ ). The evaluation of $C V a R$ requires previously the estimation of Value at Risk $(V a R)$. Here, the moments' method by Johnson has been used in order to find both $V a R$ and $C V a R$. By the way, the international financial regulators are adopting more and more a very severe quantitative analysis based on these methods in order to support financial policy choices: $\operatorname{VaR}$ and $C V a R$ are more and more appropriate tools to support financial regulatory policies.

Coming to the results, comparing the main numerical statistics and the values of the two cited measures of financial risk, $V a R$ and $C V a R$, obtained with a roll-over mechanism from January 2008 to July 2012, the impact of the crisis on equity market risk can be clearly shown. It seems that there has been a general improvement in the equity risk of all over the regions and countries: VaR and CVaR are now decreased more than half, coming back to levels strictly near the pre-crisis values except for Greece. To have some evidence of these facts, a glance must be given to the graphs reported at the end of the paper: they show VaR and CVaR with $c=99 \%$ over the whole period and over the crucial time span April 2012-July 2012 for any of the considered stock market indices.

Another important remark regards the differences between $\mathrm{VaR}$ and $\mathrm{CVaR}$ : they are not negligible and probably the influence of the crisis has increased these discrepancies given the relevance of tail-risk during the before analyzed turbulences, well captured by $C V a R$.

In the last part of our research, index of performances (expected return over risk measure) have been evaluated and compared. Starting from the well known Sharpe ratio, other indices of performance based on VaR and CVaR have been introduced. These indices, sometimes, give different answers with reference to the risk numbers 
themselves: e.g. in 2008 Malaysia and Sri Lanka have the lowest risk expected value but even so they have the lowest performance indices expected values.

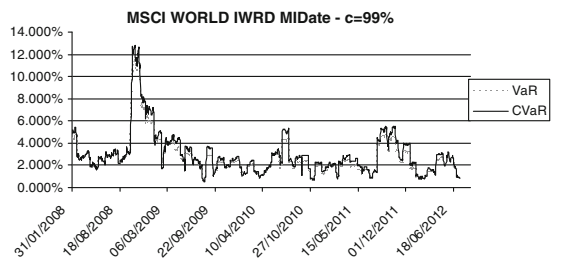

Fig. 2

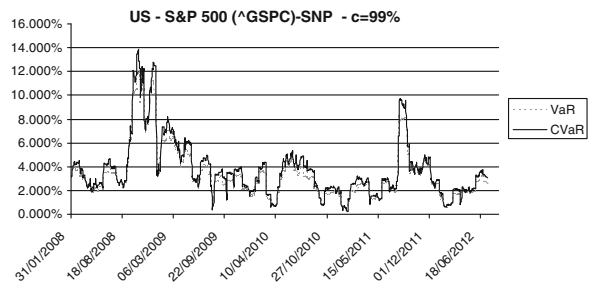

Fig. 4

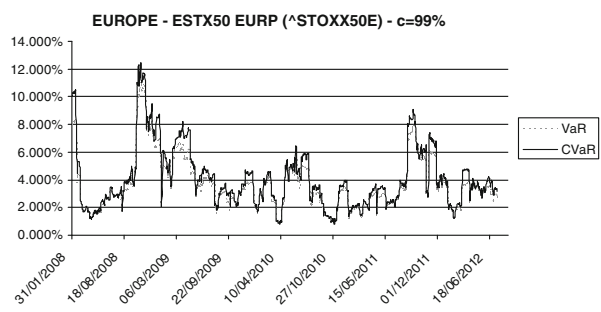

Fig. 6

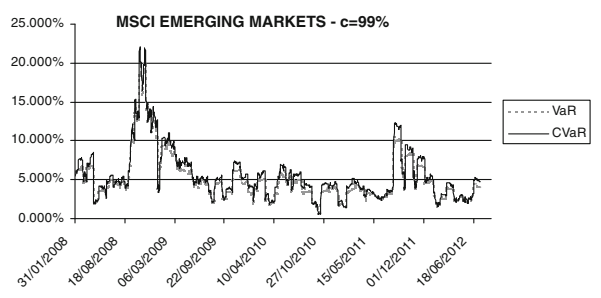

Fig. 8

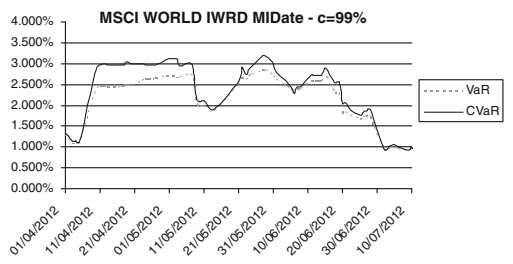

Fig. 3

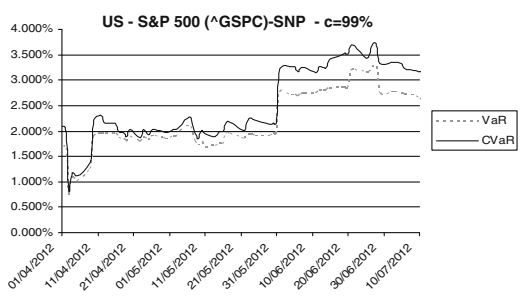

Fig. 5

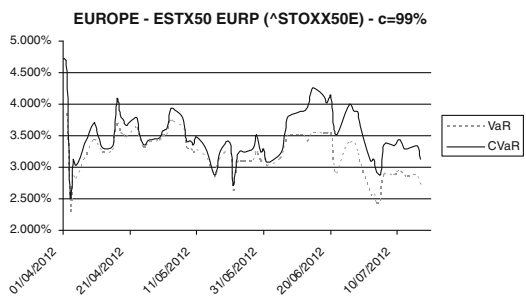

Fig. 7

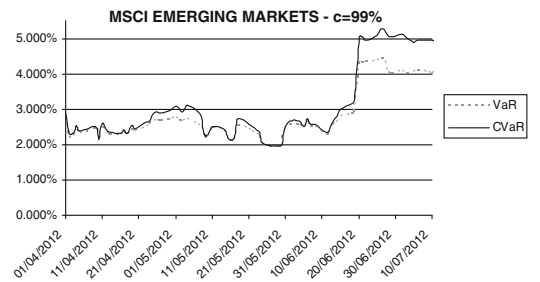

Fig. 9 


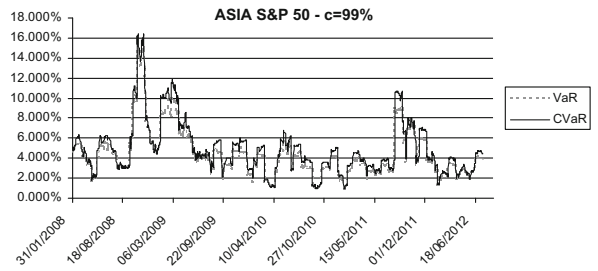

Fig. 10

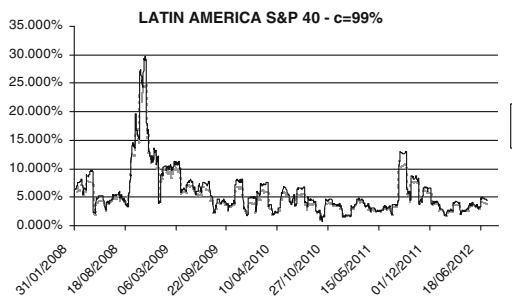

Fig. 12

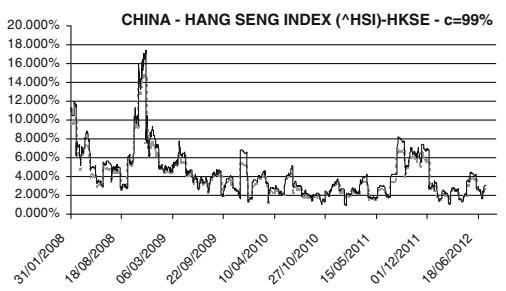

Fig. 14

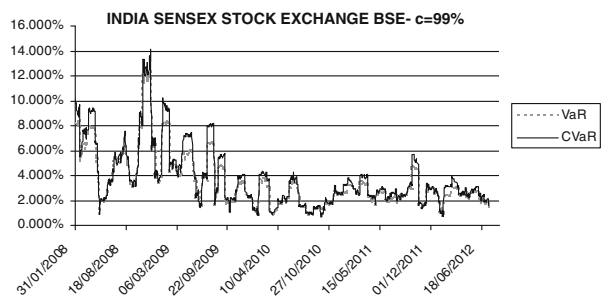

Fig.16

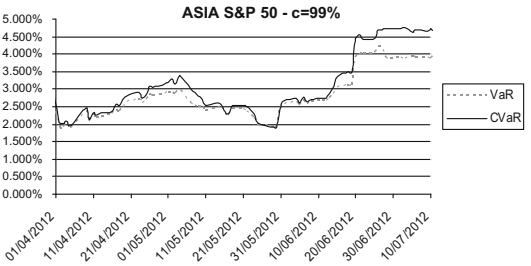

Fig. 11

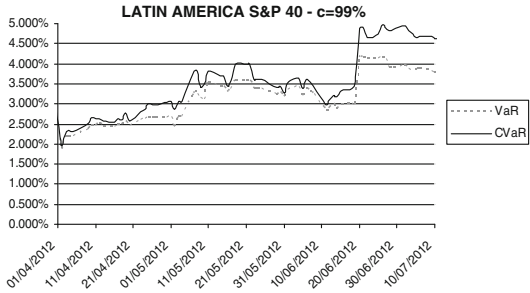

Fig. 13

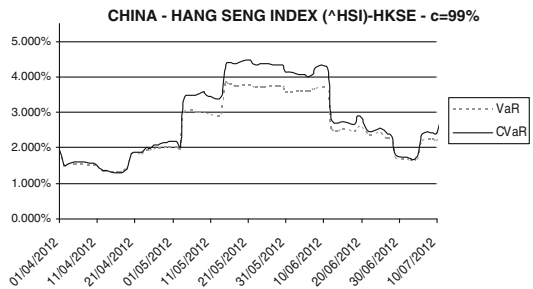

Fig. 15

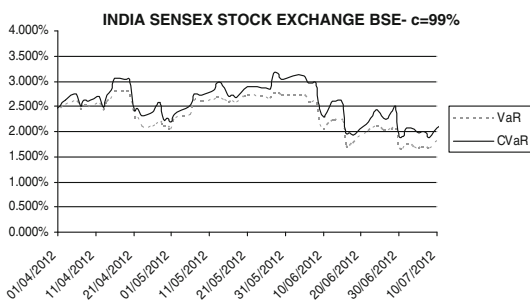

Fig. 17 


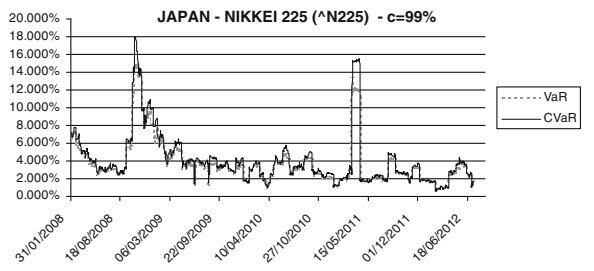

Fig. 18

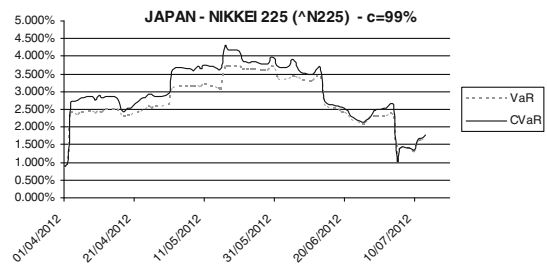

Fig. 19

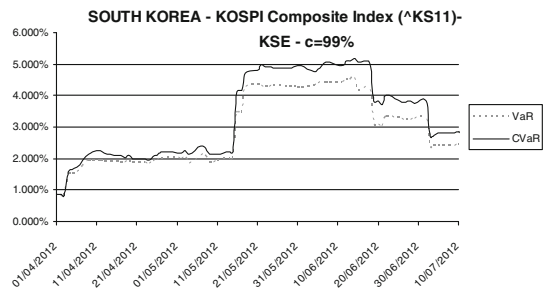

Fig. 21

Fig. 20

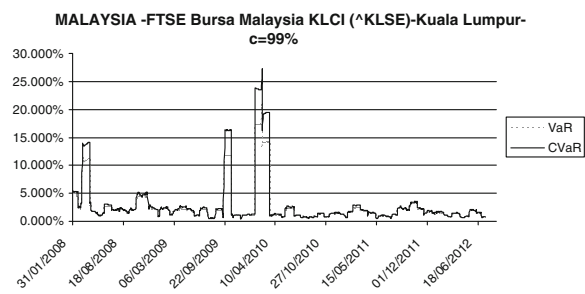

Fig. 22

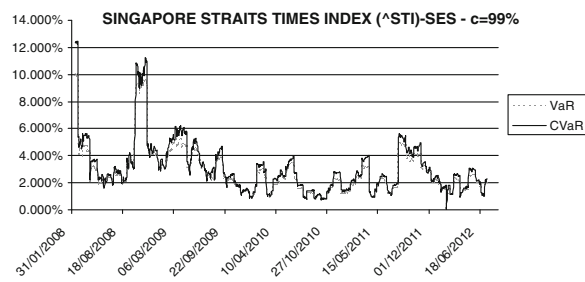

Fig. 24 KSE - $\mathrm{c}=99 \%$
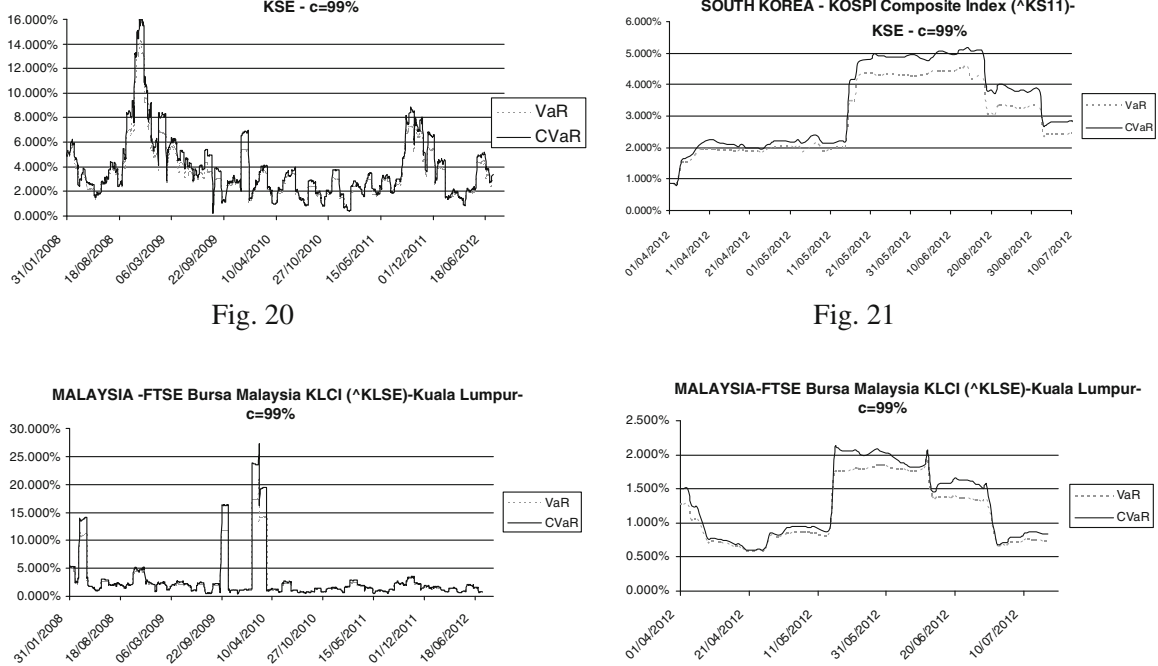

Fig. 23

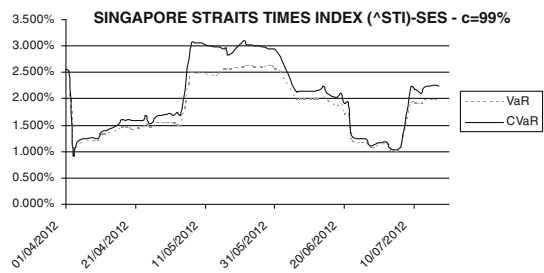

Fig. 25 


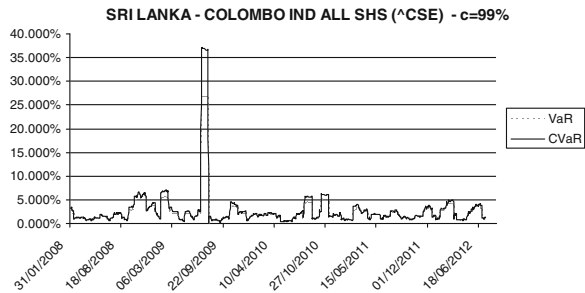

Fig. 26

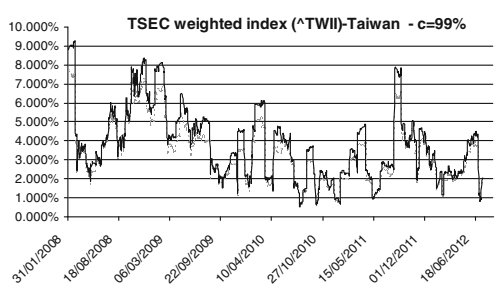

Fig. 28

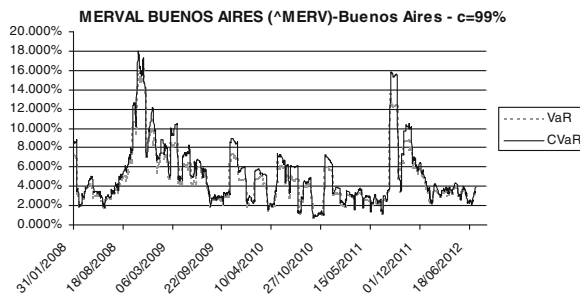

Fig. 30

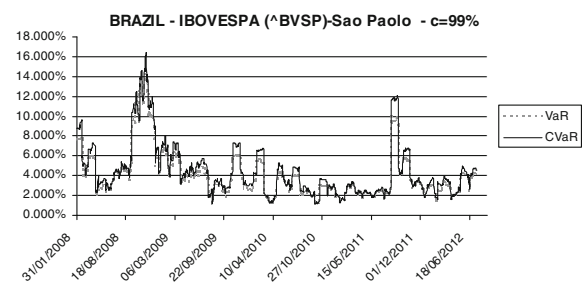

Fig. 32

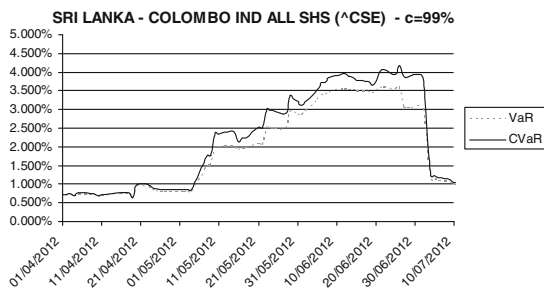

Fig. 27

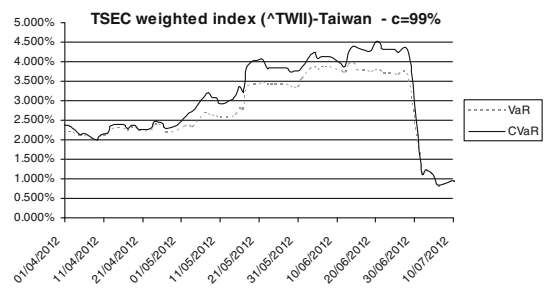

Fig. 29

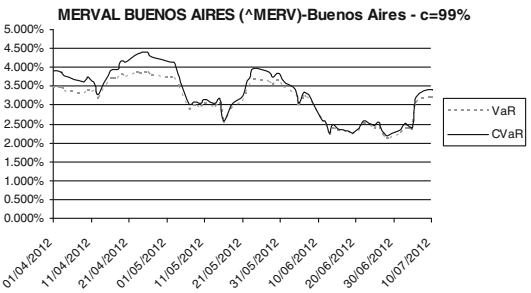

Fig. 31

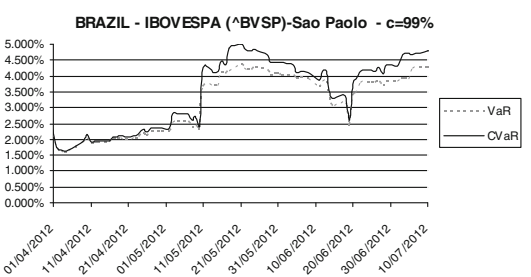

Fig. 33 


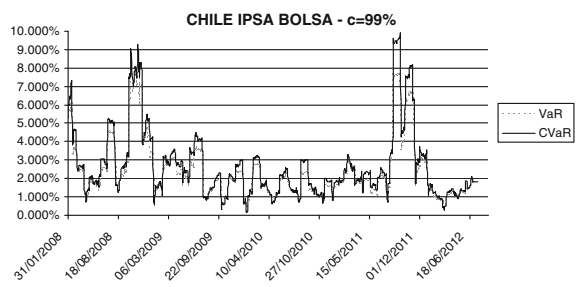

Fig. 34

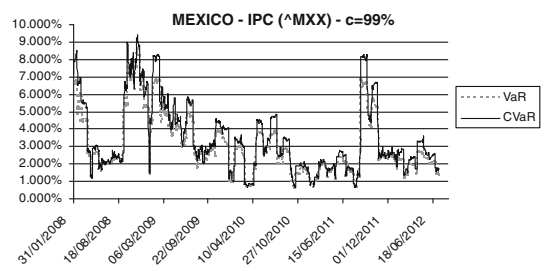

Fig. 36

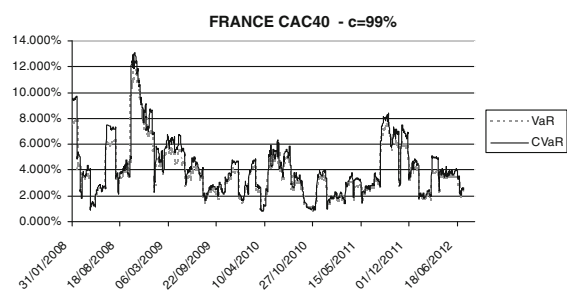

Fig. 38

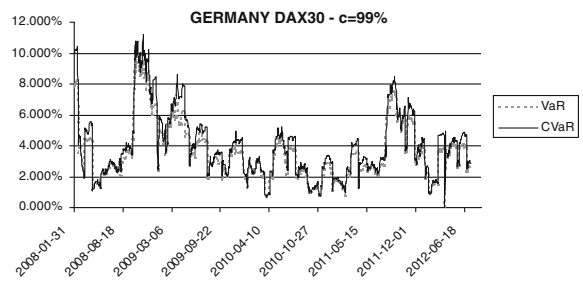

Fig. 40

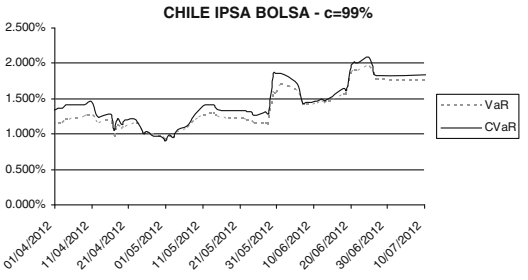

Fig. 35

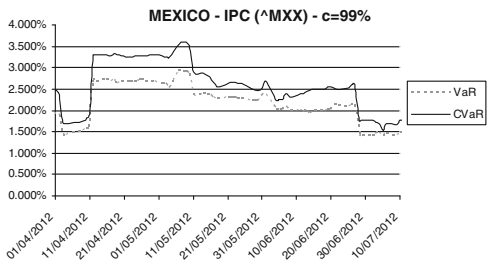

Fig. 37

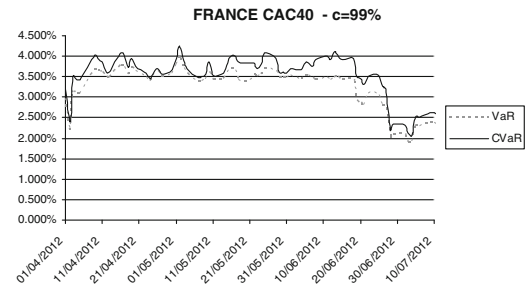

Fig. 39

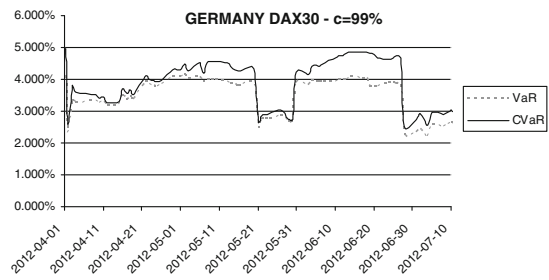

Fig. 41 
GREECE - FTSE/ATHEX 20 (FTASE.AT)-Athens - c=99\%

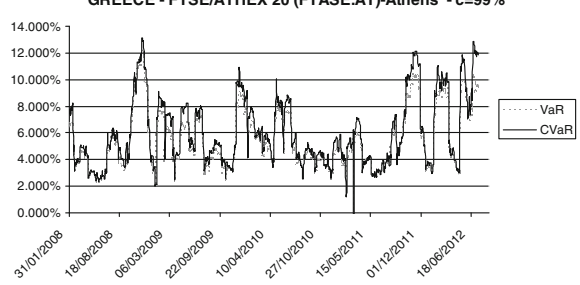

Fig. 42

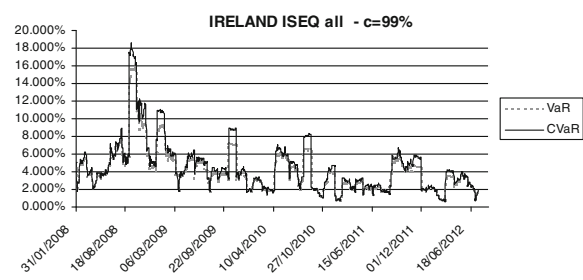

Fig. 44

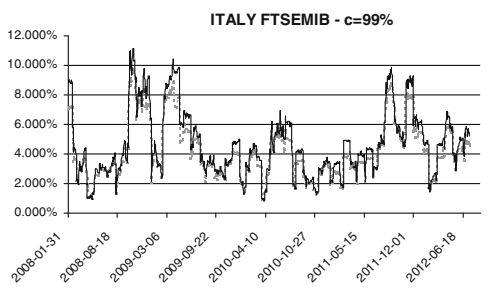

Fig. 46

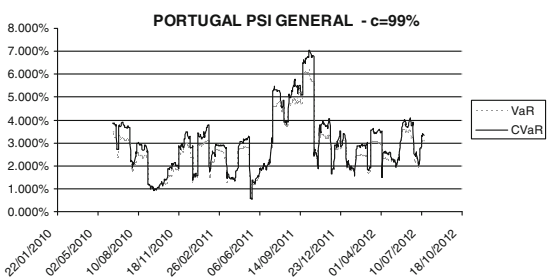

Fig. 48
GREECE - FTSE/ATHEX 20 (FTASE.AT)-Athens - C=99\%

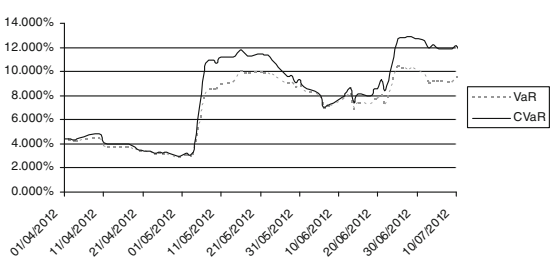

Fig. 43

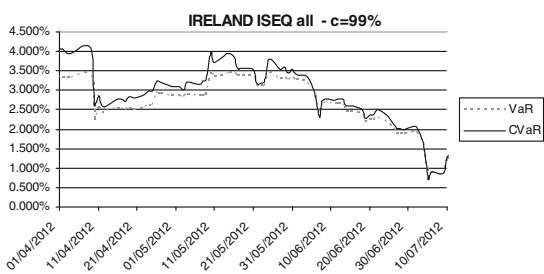

Fig. 45

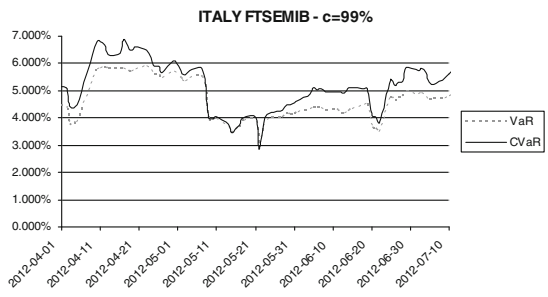

Fig. 47

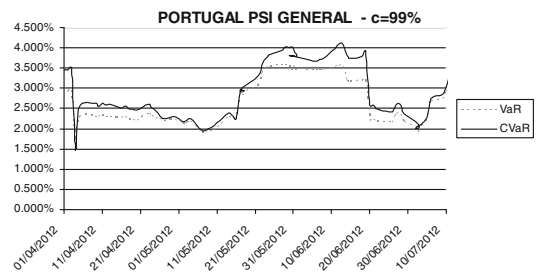

Fig. 49 


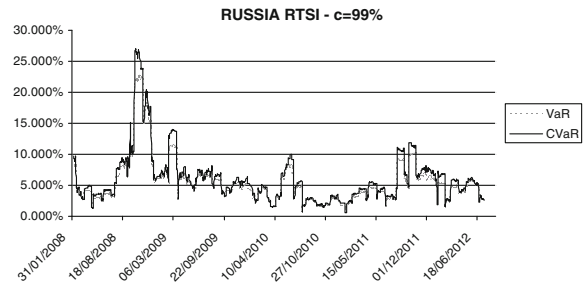

Fig. 50

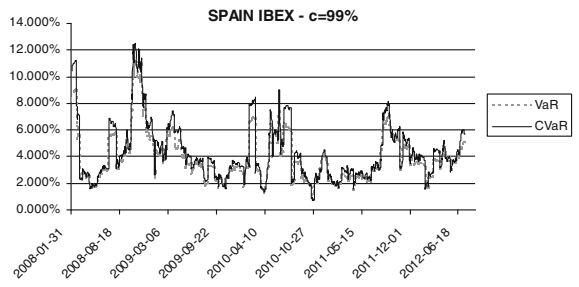

Fig. 52

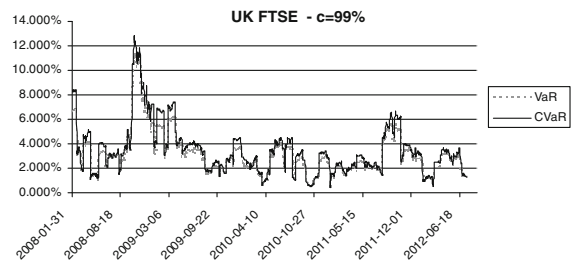

Fig. 54

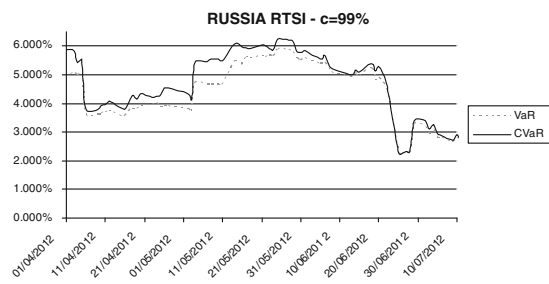

Fig. 51

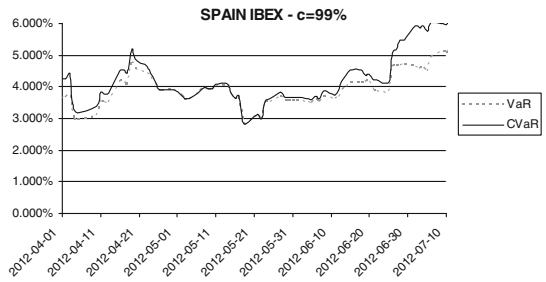

Fig. 53

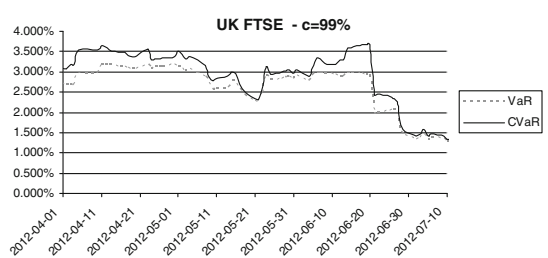

Fig. 55

\section{References}

Artzner P, Delbaen F, Eber JM, Heath D (1999) Coherent measures of risk. Math Fin 9(3):203-228

Favre L, Galeano JA (2002) Mean-modified value at risk optimization with hedge funds. J Altern Invest Fall:5

Griffiths-Hill Archive (1976) Algorithms 99 and 100. Appl Stat 25: 180 and 190, (http://lib.stat.cmu.edu/ griffiths-hill/99 and http://lib.stat.cmu.edu/griffiths-hill/100)

Hill ID, Hill R, Holder RL (1976) Algorithms AS 99. Fitting Johnson curves by moments. Appl Stat 25:180-189

Johnson NL (1949) Systems of frequency curves generated by methods of translation. Biometrika $36: 149-176$

Johnson NL (1965) Tables to facilitate fitting su frequency curves. Biometrika 52:547-558

Li XM, Rose LC (2009) The tail risk of emerging stock markets. Emerg Mark Rev 10:242-256

Rachev S (ed) (2003) Handbook of heavy tailed distributions in finance. Elsevier, Amsterdam

Shapiro SS, Slifker JF (1980) The Johnson system: selection and parameter estimation. Technometrics 22(2):239-246

Sharpe WF (1994) The Sharpe ratio. J Portfolio Manag Fall:45-58

Simonato JG (2011) The performance of Johnson distributions for computing value at risk and expected shortfall. J Deriv 19(1):7-24 